\title{
THE RINGS OF CHARIKLO UNDER CLOSE ENCOUNTERS WITH THE GIANT PLANETS
}

\author{
R. A. N. Araujo, R. Sfair, and O. C. Winter \\ UNESP - Univ. Estadual Paulista, Grupo de Dinâmica Orbital e Planetologia, CEP 12516-410, Guaratingueta, SP, Brazil; \\ ran.araujo@gmail.com,rsfair@feg.unesp.br, ocwinter@gmail.com \\ Received 2015 December 11; accepted 2016 April 5; published 2016 June 15
}

\begin{abstract}
The Centaur population is composed of minor bodies wandering between the giant planets that frequently perform close gravitational encounters with these planets, leading to a chaotic orbital evolution. Recently, the discovery of two well-defined narrow rings was announced around the Centaur 10199 Chariklo. The rings are assumed to be in the equatorial plane of Chariklo and to have circular orbits. The existence of a well-defined system of rings around a body in such a perturbed orbital region poses an interesting new problem. Are the rings of Chariklo stable when perturbed by close gravitational encounters with the giant planets? Our approach to address this question consisted of forward and backward numerical simulations of 729 clones of Chariklo, with similar initial orbits, for a period of 100 Myr. We found, on average, that each clone experiences during its lifetime more than 150 close encounters with the giant planets within one Hill radius of the planet in question. We identified some extreme close encounters that were able to significantly disrupt or disturb the rings of Chariklo. About $3 \%$ of the clones lose their rings and about $4 \%$ of the clones have their rings significantly disturbed. Therefore, our results show that in most cases (more than 90\%), the close encounters with the giant planets do not affect the stability of the rings in Chariklo-like systems. Thus, if there is an efficient mechanism that creates the rings, then these structures may be common among these kinds of Centaurs.
\end{abstract}

Key words: minor planets, asteroids: individual (10199 Chariklo) - planets and satellites: dynamical evolution and stability - planets and satellites: rings

\section{INTRODUCTION}

Among the orbits of the giant planets there is a population of small objects called Centaurs. There is no consensus on the definition of the Centaur population. According to the Minor Planet Center (MPC) of the IAU, Centaurs are celestial bodies with a perihelion beyond the orbit of Jupiter and with semimajor axes smaller than the semimajor axis of Neptune. ${ }^{1}$ Similarly, the Jet Propulsion Laboratory (JPL)/NASA defines the Centaur population as objects with semimajor axes between 5.5 and 30.1 AU. ${ }^{2}$ Duffard et al. (2014) classify Centaurs as celestial bodies with orbits mostly in the region between Jupiter and Neptune that typically cross the orbits of the giant planets. A similar definition is also found in Horner et al. (2004b).

2060 Chiron was the first observed body of this population (Kowal et al. 1979). Since then, the number of known Centaurs has grown. Currently, more than 400 objects are cataloged ${ }^{3}$, and from the flux of short period comets Horner et al. (2004a) estimated the total number of objects with a diameter $>1 \mathrm{~km}$ to be approximately 44,000 .

The Centaur 10199 Chariklo was discovered in 1997 by the Spacewatch program. ${ }^{4}$ In 2013, a stellar occultation revealed the existence of symmetric features encircling Chariklo, the second largest known Centaur. Braga-Ribas et al. (2014) showed that these structures are a system of two narrow and well-defined rings. This discovery was the first example of minor bodies within the select group of ringed objects.

In Braga-Ribas et al. (2014), the authors estimated that the rings have orbital radii of approximately $391 \mathrm{~km}$ and $405 \mathrm{~km}$, and widths of $7 \mathrm{~km}$ and $3 \mathrm{~km}$, respectively. They are assumed

\footnotetext{
MPC, www.minorplanetcenter.org/iau/lists/Unusual.html.

2 JPL, http://ssd.jpl.nasa.gov/sbdb_help.cgi?class $=$ CEN

3 MPC, www.minorplanetcenter.net/iau/lists/Centaurs.html.

4 Spacewatch Program, http://spacewatch.lpl.arizona.edu/discovery.html.
}

to be in the equatorial plane of Chariklo, with circular orbits. From the orbital positions of the rings, they also estimated the density of the central body to be $1 \mathrm{~g} \mathrm{~cm}^{-3}$. Apart from the equivalent radius of $124 \mathrm{~km}$, derived from the same stellar occultation, little information about the physical properties of Chariklo is available. Table 1 summarizes some of the physical and orbital parameters of Chariklo and its rings.

A detailed study of the orbital evolution of the Centaurs was presented by Horner et al. (2004a), where they analyzed the orbital evolution of 32 cataloged objects through numerical simulations of an ensemble of particles under the influence of the Jovian planets. They followed the particles both forward and backward in time and registered the dynamical evolution and fate of the particles. The orbital radius shows that Chariklo orbits between Saturn and Uranus, corresponding to a typical $U$ class object, i.e., those whose evolution is controlled by Uranus (Horner et al. 2003). For Chariklo, they found the half-life to be 10.3 (9.68) Myr.

The Centaur objects are transient. Therefore, a source is required to maintain a steady-state population. The idea of bodies coming from regions of the solar system beyond Neptune and populating the region between the planets is wellaccepted.

Levison \& Duncan (1997) estimated, through numerical integrations, the number of comets transiting between the inner and outer solar system originating from the Kuiper Belt as $\approx 1.2 \times 10^{7}$. Horner et al. (2004a) estimated a flux of one body coming from the Kuiper Belt and joining the Centaur population every 125 years. Sisto \& Brunini (2007), present the scattered disk objects (SDO; bodies with a distance to the perihelion of $q<30 \mathrm{AU}$ and semimajor axes of $a>50 \mathrm{AU}$ ) as the most probable source of the Centaurs. Emel'Yanenko et al. (2007), analyzed the role of the Oort cloud in determining the flux of cometary bodies through the planetary system. They 
Table 1

Orbital and Physical Parameters of Chariklo and its Rings

\begin{tabular}{|c|c|c|c|c|}
\hline \multicolumn{2}{|c|}{ Chariklo } & \multicolumn{3}{|c|}{ Rings } \\
\hline$a^{\mathrm{a}}$ & $15.74 \mathrm{AU}$ & & $R 1$ & $R 2$ \\
\hline$e^{\mathrm{a}}$ & 0.171 & Orbital radius $(\mathrm{km})^{\mathrm{b}}$ & $390.6 \pm 3.3$ & $404.8 \pm 3.3$ \\
\hline$i^{\mathrm{a}}$ & $23^{\circ} .4$ & Width $(\mathrm{km})^{\mathrm{b}}$ & $7.17 \pm 0.14$ & $3.4_{-1.4}^{+1.1}$ \\
\hline Equivalent radius $(\mathrm{km})^{\mathrm{b}}$ & 124 & Radial separation $(\mathrm{km})^{\mathrm{b}}$ & & \\
\hline Mass $(\mathrm{kg})^{\mathrm{c}}$ & $7.986 \times 10^{18}$ & Gap between rings $(\mathrm{km})^{\mathrm{b}}$ & & \\
\hline
\end{tabular}

Notes.

${ }^{a}$ Orbital elements obtained from JPL's Horizons system for the epoch MJD 56541. According to JPL the uncertainties in $a, e$, and $i$ are of the order $10^{-5}, 10^{-6}$, and $10^{-5}$, respectively.

${ }^{\mathrm{b}}$ Braga-Ribas et al. (2014).

${ }^{\mathrm{c}}$ Calculated considering a density of $1 \mathrm{~g} \mathrm{~cm}^{-3}$, the equivalent radius of Chariklo and a spherical body.

concluded that a substantial fraction of all known cometary bodies may have a source in the Oort cloud, including the Centaur population, which they defined as the population of small bodies with a perihelion $5<q<28 \mathrm{AU}$ and $a<1000$ AU. Following the same definition for Centaurs, Emel'Yanenko et al. (2013) suggest that more than $90 \%$ of all Centaurs with $a>60 \mathrm{AU}$ and $\approx 50 \%$ with $a<60 \mathrm{AU}$ come from the Oort cloud. In Brasser et al. (2012), the Oort cloud is also indicated out as the source of Centaurs, in particular those with high inclination. In that work the Centaur population is defined as small bodies with a perihelion between 15 and $30 \mathrm{AU}$ and a semimajor axis shorter than $100 \mathrm{AU}$. They showed that these objects probably originated from the Oort cloud rather than the Kuiper Belt or the scattered disc.

Throughout its orbital evolution a Centaur is strongly perturbed by the giant planets. Horner et al. (2004b), illustrate in detail the effects of these perturbations on the orbit of five selected Centaurs. The close encounters with the giant planets are quite frequent. As consequence, the Centaurs present a characteristic chaotic orbital evolution.

The existence of a small body with a well-defined system of circular rings within such a perturbed population poses an interesting new problem. This scenario has motivated the development of the present work. We investigated the stability of the rings of Chariklo when perturbed by close encounters with the giant planets. We analyzed how effective the close encounters are at disturbing or disrupting the rings of Chariklo. Furthermore, the development of this study may allow us to quantitatively evaluate how propitious the region of the Centaurs is for such small bodies with their own systems of rings. A brief qualitative discussion on this subject is presented by Ortiz et al. (2015), where it is proposed that the Centaur Chiron may also have rings. The possible existence of two small bodies with systems of rings belonging to the same population is quite interesting, and indicates that such systems may be more frequent than expected.

Since our goal is to analyze the stability of the rings of Chariklo when they are perturbed by close encounters with the giant planets, here we classify an object as a Centaur when its orbit is mainly in the region between Jupiter and Neptune (as in Duffard et al. 2014), with a maximum semimajor axis value of $a \leqslant 50$ AU.

The structure of this paper is as follows. In Section 2, we present the initial conditions and the numerical method adopted in order to identify the close encounters of Chariklo with the giant planets. In Section 3, we present the selection of the extreme close encounters, i.e., those encounters that could be capable of disrupting the rings of Chariklo. In Section 4, we describe how the rings were simulated during a close encounter. In Sections 5-7 we present the results, and Section 8 provides our final comments and the major conclusions of the work.

\section{CLOSE ENCOUNTERS WITH THE GIANT PLANETS}

The first step consisted on selecting a representative sample of close encounters of Chariklo with the giant planets. We considered a system composed of the Sun, the giant planets of the solar system (Jupiter, Saturn, Uranus, and Neptune), and a sample of clones, i.e., objects with the same mass and radius as Chariklo, but with small deviations in their orbits.

The clones were created following the procedure presented in Horner et al. (2004a), where 729 clones were created from the original orbit assuming a variation of semimajor axis of $0.005 \mathrm{AU}$, a variation of eccentricity of 0.005 , and a variation of inclination of 0.01 .

The orbital elements of Chariklo and of the planets were obtained through JPL's Horizons system for the epoch MJD 56541. For the orbit of Chariklo at this epoch we have $a=$ 15.74 AU, $e=0.171$, and $i=23^{\circ} .4$.

Considering these orbital elements, and taking the amplitude of variation as in Horner et al. (2004a) in such way that we have 729 clones, we created the clones of Chariklo orbiting the Sun as follows: $15.720 \leqslant a \leqslant 15.760 \mathrm{AU}$, taken every $0.005 \mathrm{AU} ; 0.151 \leqslant e \leqslant 0.191$, taken every 0.005 ; and $23^{\circ} .36 \leqslant$ $i \leqslant 23,44^{\circ}$, taken every $0^{\circ} 01$. The choice of these values resulted in nine values of semimajor axes, nine values of eccentricities, and nine values of inclination. The combination of these values resulted in 729 clones, each one with different values of $a, e$, and $i$. Considering that Chariklo has an equivalent radius of $124 \mathrm{~km}$ and a density of $1 \mathrm{~g} \mathrm{~cm}^{-3}$ (Braga-Ribas et al. 2014), we estimated its mass as $M_{C}=7.986 \times 10^{18} \mathrm{~kg}$. We performed backward and forward numerical integrations of the system composed of the Sun, the giant planets, and the clones, for a time span of $100 \mathrm{Myr}$, using the adaptive time-step hybrid sympletic/Bulirsch-Stoer algorithm from MERCURY (Chambers 1999).

Throughout the numerical integrations the clones did not interact with each other, but they could collide with the planets or escape from the system. The collisions were defined by the relative distance between the clones and the planets. If the clone-planet distance was smaller than the radius of the planet 


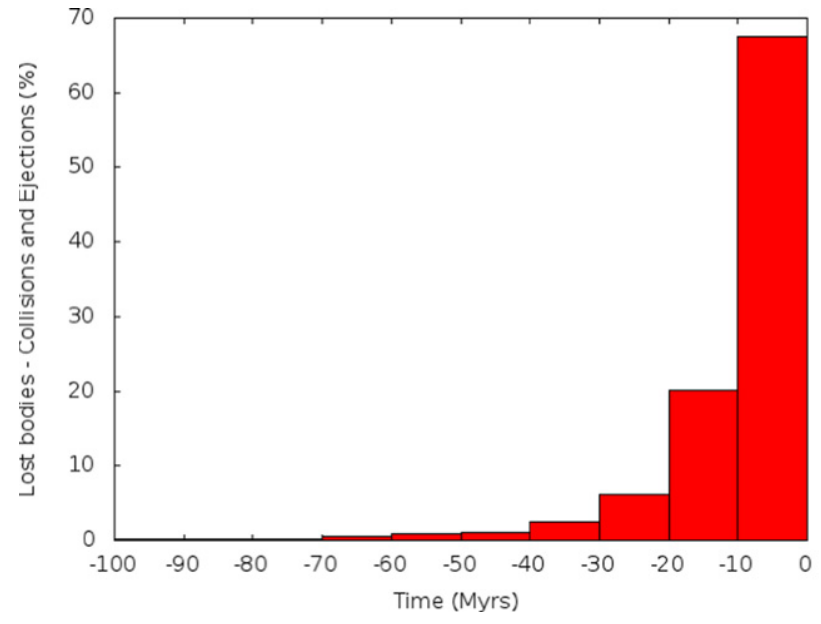

(a)

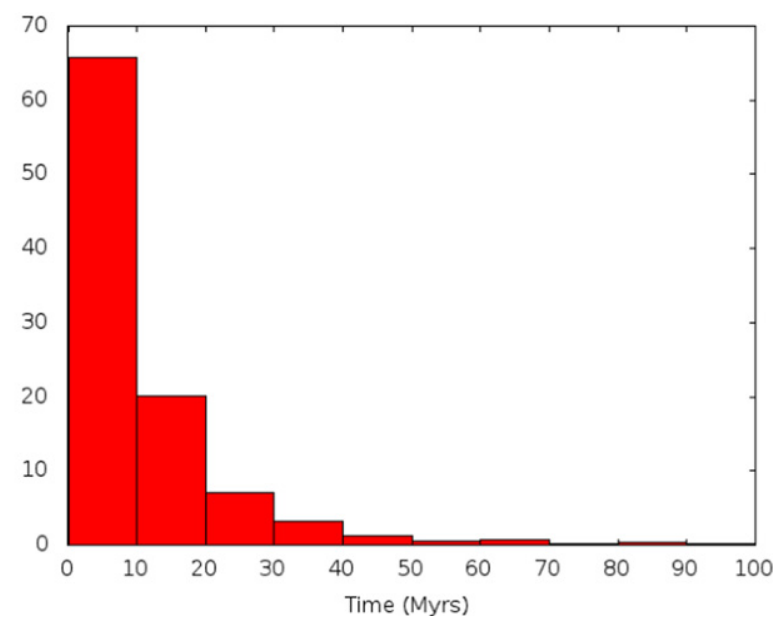

(b)

Figure 1. Histograms of the fraction of Chariklo clones lost within $100 \mathrm{Myr}$ as a function of time. (a) Backward integration. (b) Forward integration. Throughout the numerical integration the clones could be lost by ejection or collisions with one of the giant planets or with the Sun. The ejection distance was considered to be $100 \mathrm{AU}$ and the collisions were defined by the physical radius of the planets and of the Sun.

Table 2

Registered Close Encounters of the Clones with Each of the Giant Planets within 1 Hill Radius and within 1 and 10 Rupture Radii $\left(r_{\mathrm{td}}\right)$ in the Time Span of $10 \mathrm{Myr}$, for both Forward and Backward Integrations

\begin{tabular}{|c|c|c|c|c|c|c|c|c|}
\hline \multirow{2}{*}{ Planet } & \multirow{2}{*}{$\begin{array}{l}\text { Hill Radius }^{\mathrm{a}} \\
\text { (Planetary } \\
\text { Radius) }\end{array}$} & \multirow{2}{*}{$\begin{array}{c}r_{\mathrm{td}}^{\mathrm{a}} \\
\text { (Planetary } \\
\text { Radius) }\end{array}$} & \multicolumn{3}{|c|}{ Forward Encounters } & \multicolumn{3}{|c|}{ Backward Encounters } \\
\hline & & & $\begin{array}{l}1 \text { Hill } \\
\text { Radius }\end{array}$ & $r_{\mathrm{td}} \leqslant 1$ & $1<r_{\mathrm{td}} \leqslant 10$ & $\begin{array}{c}1 \text { Hill } \\
\text { Radius }^{c}\end{array}$ & $r_{\mathrm{td}} \leqslant 1$ & $1<r_{\mathrm{td}} \leqslant 10$ \\
\hline Jupiter & $\approx 740$ & $\approx 5$ & $16.6 \%$ & 5 & 36 & $18.3 \%$ & 5 & 47 \\
\hline Saturn & $\approx 1100$ & $\approx 4$ & $26.0 \%$ & 1 & 34 & $24.2 \%$ & 0 & 25 \\
\hline Uranus & $\approx 2700$ & $\approx 5$ & $48.0 \%$ & 0 & 18 & $46.9 \%$ & 2 & 13 \\
\hline Neptune & $\approx 4600$ & $\approx 5$ & $9.4 \%$ & 0 & 2 & $10.6 \%$ & 1 & 5 \\
\hline
\end{tabular}

Notes.

a The Hill radius and the rupture radius in terms of the radius of the planet in question.

${ }^{\mathrm{b}}$ Percentage relative to 60159 encounters.

${ }^{c}$ Percentage relative to 65293 encounters.

in question, then we had a collision. The physical radius of the planet was determined by Mercury, assuming a spherical planet with uniform density. We consider ejections as being the ejections from the Centaur population defined by the relative distance to the Sun of $100 \mathrm{AU}$. This value was adopted taking into account that if a clone reached the distance of $100 \mathrm{AU}$ and was still in a elliptical orbit, then necessarily the semimajor axis of the clone had to be greater than $50 \mathrm{AU}$, i.e., the clone was no longer classified as a Centaur, according to our definition.

As a result of the integrations, we see in the histograms in Figure 1 that more than $50 \%$ of our sample was lost (ejections or collisions) in $10 \mathrm{Myr}$, for both backward and forward integrations. These results show that the evolution of our sample is in agreement with the predicted evolution of the Centaurs, which have an estimated mean lifetime of about $10 \mathrm{Myr}$ (Tiscareno \& Malhotra 2003). We also note that there is a kind of symmetry in the results, which indicates that Chariklo is currently in the middle of its median dynamical lifetime as a Centaur.

At the end of the forward integrations, we found that $\approx 94 \%$ of the 729 clones were lost in the time span of $100 \mathrm{Myr}, 683$ clones being lost through ejections and four clones through collisions (three with Saturn and one with Jupiter). For the backward integration, we found that $\approx 99 \%$ of the clones were lost in $100 \mathrm{Myr}$, with 719 ejections and four collisions (three with Jupiter and one with Saturn).

Once we characterized the evolution of the sample of clones as a whole, we then selected all close encounters of the clones within 1 Hill radius with each giant planet occurring within $10 \mathrm{Myr}$ (the mean lifetime of the Centaurs). For this time span, 60159 close encounters were registered for the forward integration and 65293 encounters for the backward integration. From Table 2 we see that in this case Uranus dominates, followed by Saturn, Jupiter, and Neptune, for both the backward and forward integrations. This result is in agreement with works on the dynamics of Centaurs, which state that the dynamics of bodies with similar orbits to the orbit of Chariklo should be guided by Uranus, as discussed in Horner et al. (2004a).

However, we are interested in analyzing how the close encounters of Chariklo with the giant planets might affect its rings. Therefore, we selected from among all the registered close encounters those that are expected to perturb or disrupt the rings. The details of this analysis and the results obtained are described in the following.

\section{EXTREME CLOSE ENCOUNTERS}

In order to select the extreme close encounters, i.e., encounters with the giant planets that are expected to 
significantly affect the rings of Chariklo, we calculated the tidal disruption radius $\left(r_{\mathrm{td}}\right)$. According to Philpott et al. (2010), the distance of a close encounter at which the tidal disruption of a binary may occur is given by:

$$
r_{\mathrm{td}} \approx a_{B}\left(\frac{3 M_{\mathrm{Pl}}}{M_{1}+M_{2}}\right)^{1 / 3}
$$

where $a_{B}$ is the semimajor axis of the binary, $M_{\mathrm{Pl}}$ is the mass of the planet, and $M_{1}$ and $M_{2}$ are the masses of the components of the binary.

For a particle orbiting Chariklo with $a_{B}=410 \mathrm{~km}$ (the approximate outer limit of the ring), we calculated the $r_{\mathrm{td}}$ for encounters with each one of the giant planets. These values are presented in Table 2. It is important to point out that this is an approximate value since it does not take into account the relative velocity of the bodies at the moment of the encounter. Araujo et al. (2008) showed that not just the distance of the encounter, but also the relative encounter velocity determine how significantly a body will be disturbed by a close encounter. Such effects were also discussed by Araujo \& Winter (2014) when they compared their numerical analysis of the disruption of Near-Earth Asteroid (NEA) binaries due to close encounters with the Earth with the analytical prediction given by Equation (1), showing the dependence of the results on the relative velocity of the encounters.

Nevertheless, for our purposes the approach given by Equation (1) is adequate. Knowing that this value is an approximation, we then selected from all the registered close encounters those that had a minimum distance within $10 r_{\mathrm{td}}$. For the forward integration, we see that most of these (about 3/4) occurred with Jupiter and Saturn (Table 2). Very few encounters occurred within $1 r_{\mathrm{td}}$ (none with Uranus or Neptune). For the backward integration, we see that the extreme encounters with Jupiter and Saturn still prevail, but here we note the occurrence of a few encounters within $1 r_{\mathrm{td}}$ with Uranus and Neptune. We explored the effects of each of these extreme encounters $\left(\leqslant 10 r_{\mathrm{td}}\right)$ on the particles of Chariklo's rings, as described in the following.

\section{SIMULATING THE RINGS}

As the second step of our study, we numerically simulated the extreme close encounters, including massless particles around Chariklo. According to Section 2, a close encounter is registered when a clone of Chariklo crosses the limit of 1 Hill radius of any of the giant planets. At this crossing moment, we recorded the position and the velocity of these bodies relative to the Sun. These values are the initial conditions for the simulations of the extreme close encounters, i.e., encounters with minimum distance within $10 r_{\mathrm{td}}$. Thus, at this step, the numerical simulations always involve the Sun, the bodies performing the close encounter (the planet and Chariklo), and a sample of particles orbiting Chariklo.

We considered particles with circular equatorial orbits, radially distributed from $200 \mathrm{~km}$ to $1000 \mathrm{~km}$, taken every $20 \mathrm{~km}$. For each radial distance 100 particles were considered in a random angular distribution. Such a combination of values resulted in a total of 4100 particles orbiting Chariklo.

The pole orientation of Chariklo was considered to be perpendicular to the orbital plane. This is a reasonable approach since the rings are assumed to be in the equatorial plane of Chariklo, and we are interested in the maximized radial perturbations of the rings.

The encounters were simulated for a time span of 1 year using the adaptive time-step Gauss-Radau numerical integrator, keeping the accuracy at $10^{-12}$ (Everhart 1985, pp. 185-202). Throughout the integration, the particles could collide with Chariklo or be ejected. The collisions were defined by the equivalent radius of Chariklo $(124 \mathrm{~km})$. The ejections were defined by the energy of the two-body problem Chariklo particle.

According to Table 2, 96 close encounters were simulated for the forward case and 98 for the backward case. The results of these simulations and their implications are presented in Table 3 and they are discussed in the following sections.

\section{CATASTROPHIC ENCOUNTERS}

We classify as catastrophic those close encounters that led to the complete removal of the particles in the region of the rings of Chariklo. Knowing that the particles of the rings are distributed in the range of $\approx 390 \mathrm{~km}$ to $\approx 405 \mathrm{~km}$, we defined that there was a catastrophic encounter if at the end of our simulation particles distributed beyond $380 \mathrm{~km}$ were lost by ejection or collision as defined in Section 4. The results presented in Table 3 show that in about $10 \%$ of the simulations the rings were removed from Chariklo due to close gravitational encounters with the giant planets, for both the backward and forward integrations.

For the forward integration, we found that only extreme encounters with Jupiter and Saturn were able to fully remove the rings. For the backward integration there were a few cases where Uranus and Neptune were able to do so. Our data suggest that Uranus and Neptune might have influenced the existence of the rings in the past, but from now on Jupiter and Saturn would play this role. However, more simulations are required to investigate whether this is a real difference in the forward/backward evolution or the result of a statistical artifact due to the small number of extreme close encounters.

In the cases of forward integration in time, after the removal of the rings, Chariklo remained as a Centaur for much less than a million years, i.e., the rings were destroyed in the very last stage of Chariklo's orbital evolution among the giant planets. In the cases of backward integration in time, the removal of the rings occurred long before Chariklo completed its first million years as a Centaur, i.e., in its first stage of orbital evolution among the giant planets.

\section{DISTURBING ENCOUNTERS}

There were cases in which the particles of the rings were not removed, but their orbits were significantly changed due to a significant perturbation caused by an encounter with a giant planet. In Figure 2, we present an example of the effects of such an encounter with Jupiter. The plots show the maximum final variation in semimajor axis and eccentricity among the hundred particles that share the same initial radial position. As expected, the values increase with the radial distance. In the region of the rings (indicated by the vertical lines) the semimajor axis changes by more than $30 \mathrm{~km}$ and the eccentricity grows up to more than 0.5.

The results of the encounters that produced at least some noticeable variations $(\Delta e \geqslant 0.01)$ on the orbits of the rings are 
Table 3

Registered Catastrophic and Disturbing Encounters of the Rings of Chariklo due to Extreme Close Encounters with Each of the Giant Planets over a Time Span of $10 \mathrm{Myr}$

\begin{tabular}{|c|c|c|c|c|c|c|}
\hline & \multicolumn{3}{|c|}{ Forward Encounters } & \multicolumn{3}{|c|}{ Backward Encounters } \\
\hline & Catastrophic $^{\mathrm{a}}$ & Disturbing $^{\mathrm{b}}$ & $\begin{array}{c}\text { Survival Time }{ }^{\mathrm{c}} \\
\text { [Max:Min](years) }\end{array}$ & Catastrophic $^{\mathrm{a}}$ & Disturbing $^{\mathrm{b}}$ & $\begin{array}{l}\text { Survival Time }{ }^{\mathrm{c}} \\
\text { [Max:Min](years) }\end{array}$ \\
\hline Saturn & 4 & 7 & {$[56,650: 623,559]$} & 0 & 7 & $\ldots$ \\
\hline Uranus & 0 & 0 & $\ldots$ & 3 & 1 & {$[-1,371,579:-4,401,849]$} \\
\hline Neptune & 0 & 0 & $\ldots$ & 1 & 2 & $-1,499,269$ \\
\hline
\end{tabular}

Notes.

${ }^{a}$ Rings are completely removed.

${ }^{\mathrm{b}}$ External particles removed but the rings survive.

${ }^{c}$ Minimum and maximum survival time registered among the clones after they suffered a catastrophic encounter, excluding the immediate ejection cases.

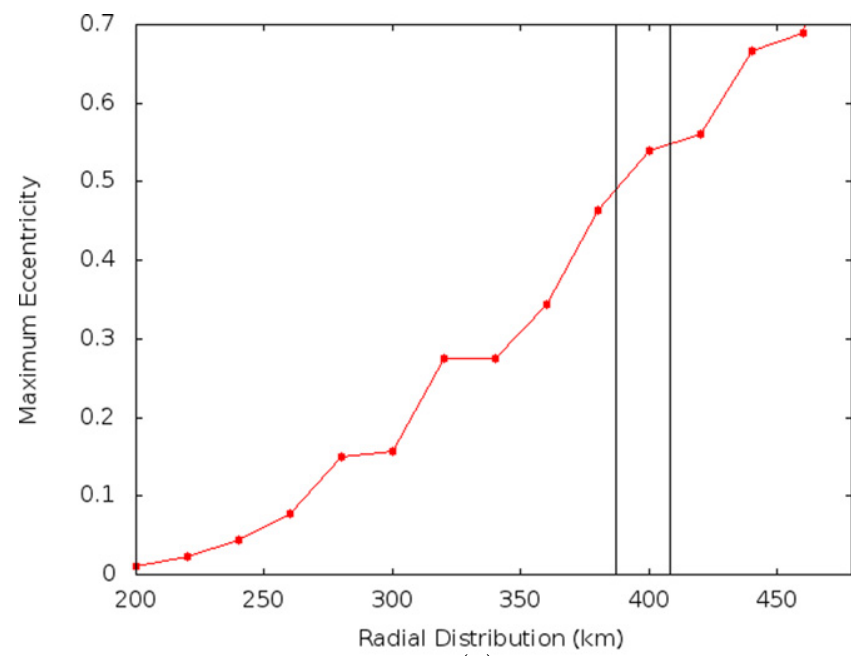

(a)

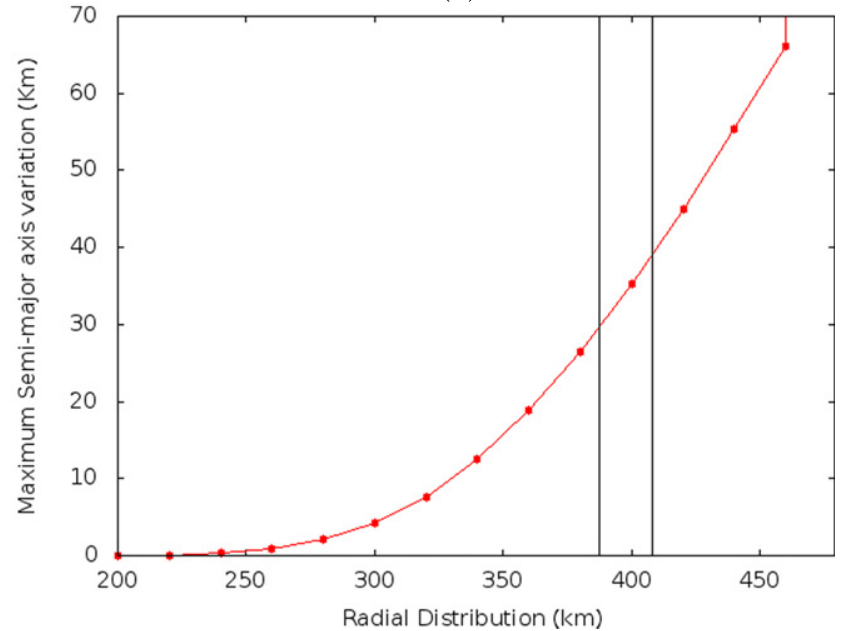

(b)

Figure 2. An example of a disturbing close encounter caused on the ring by a close encounter with Jupiter. (a) Maximum final change in eccentricity. (b) Maximum final change in semimajor axis $(\mathrm{km})$. The plots show the maximum final changes among the hundred particles that share the same initial radial position. The vertical lines indicate the boundary of the ring region. The encounter was performed with a minimum distance of 6.6 planet radii, with a relative velocity of $V_{\infty}=8.00 \mathrm{~km} \mathrm{~s}^{-1}$. The encounter resulted in a critical radius of $R_{C}=460 \mathrm{~km}$, meaning that no particles in the region of the rings were removed.
Table 4

List of Significant Disturbing Encounters $(\Delta e \geqslant 0.01)$ with the Giant Planets for both Forward and Backward Integration within the Time Span of $10 \mathrm{Myr}$

\begin{tabular}{|c|c|c|c|c|}
\hline $\begin{array}{l}\text { Minimum Encounter } \\
\text { Distance } \\
\text { (Planet Radius) }^{\mathrm{a}}\end{array}$ & $\begin{array}{r}\begin{array}{r}V_{\infty} \\
\left(\mathrm{km} \mathrm{s}^{-1}\right)\end{array} \\
\text { Jupi }\end{array}$ & $\begin{array}{l}\text { Maximum } \\
\text { Ring } \\
\Delta a(\mathrm{~km}) \\
\text {-Forward }\end{array}$ & $\begin{array}{c}\text { Maximum } \\
\text { Ring } \\
\Delta e\end{array}$ & $\begin{array}{c}\text { Survival } \\
\text { Time }^{\mathrm{b}} \\
\text { (years) }\end{array}$ \\
\hline 6.6 & 4.00 & 35.28 & 0.54 & 451,203 \\
\hline 6.9 & 4.67 & 7.61 & 0.28 & 509,333 \\
\hline 8.5 & 5.81 & 34.40 & 0.45 & 43,868 \\
\hline 8.8 & 3.76 & 28.20 & 0.32 & 6891 \\
\hline 9.2 & 5.03 & 44.33 & 0.57 & $1,140,000$ \\
\hline 15.4 & 7.00 & 0.04 & 0.02 & 55,034 \\
\hline 16.0 & 7.78 & 0.02 & 0.01 & 59,483 \\
\hline \multicolumn{5}{|c|}{ Saturn-Forward } \\
\hline 6.8 & 4.87 & 24.77 & 0.34 & 22,115 \\
\hline 7.4 & 3.12 & 30.13 & 0.53 & 337,731 \\
\hline 8.2 & 4.42 & 1.23 & 0.07 & 87,594 \\
\hline 9.3 & 5.29 & 0.68 & 0.06 & 253,520 \\
\hline 10.4 & 3.63 & 0.14 & 0.031 & 573,463 \\
\hline \multicolumn{5}{|c|}{ Jupiter-Backward } \\
\hline 4.2 & 9.87 & 25.59 & 0.20 & $-217,796$ \\
\hline 8.3 & 4.17 & 17.34 & 0.40 & $-18,259$ \\
\hline 8.4 & 4.96 & 13.63 & 0.33 & $-23,770$ \\
\hline 8.4 & 10.32 & 65.85 & 0.51 & $-8,204$ \\
\hline 8.5 & 7.23 & 2.42 & 0.20 & $-280,371$ \\
\hline 11.1 & 4.93 & 0.065 & 0.03 & $-84,066$ \\
\hline 11.1 & 5.20 & 5.74 & 0.19 & $-153,800$ \\
\hline 11.9 & 6.62 & 2.33 & 0.10 & $-537,024$ \\
\hline 13.4 & 3.17 & 0.28 & 0.04 & $-309,943$ \\
\hline 13.5 & 7.40 & 0.06 & 0.02 & $-36,896$ \\
\hline 14.4 & 1.25 & 0.03 & 0.01 & $-98,210$ \\
\hline 15.4 & 5.72 & 0.02 & 0.01 & $-45,522$ \\
\hline 16.7 & 9.65 & 0.03 & 0.01 & $-1,634,011$ \\
\hline \multicolumn{5}{|c|}{ Saturn-Backward } \\
\hline 7.9 & 2.68 & 13.79 & 0.300 & $-405,570$ \\
\hline \multicolumn{5}{|c|}{ Uranus_-Backward } \\
\hline 10.8 & 3.00 & 3.73 & 0.11 & $-2,504,843$ \\
\hline
\end{tabular}

Notes. The immediate ejection cases were excluded.

${ }^{\text {a }}$ The minimum encounter distance given in terms of the radius of the planet in question.

${ }^{\mathrm{b}}$ Survival time after a disturbing encounter. 
presented in Table 4 for backward and forward integrations, excluding the encounters followed by ejection.

For the forward integration, we observed only five encounters with Jupiter and two with Saturn that resulted in eccentricities larger than 0.1 for the ring particles. None of the encounters with Uranus or Neptune showed significant orbital variation of the particles of the rings. Similarly, if we look backward, we see fewer close encounters that could have increased the eccentricity of the particles of the rings for values larger than 0.1 (five with Jupiter, one with Saturn, and one with Uranus).

Since Chariklo, as observed now, has well-defined narrow circular rings (Braga-Ribas et al. 2014), this might mean that it did not suffer any of these disturbing encounters or, if they did occur, it was so long ago that the rings had time to evolve, damping the eccentricity and reshaping the rings. Both possibilities are compatible with our results and, as shown in Table 3, the probability of catastrophic or disturbing encounters is very low, and probably occurred several million years ago.

\section{THE EJECTION CASES}

The close encounters of Chariklo with any of the giant planets followed by ejections are particularly interesting. If we look forward, these would be the encounters responsible for removing Chariklo from the population of Centaurs. On the other hand, looking backward, these events would be the ones that brought Chariklo into the region that we define as the Centaur population (see Section 1). We then looked for the last close encounter (within 1 Hill radius) that each clone suffered before being ejected. For the forward integration, we found that approximately $36 \%$ of the clones suffered an encounter with Jupiter before the ejection, $45 \%$ with Saturn, $10 \%$ with Neptune, and $9 \%$ with Uranus. For the backward integration, we found that approximately $27 \%$ of the encounters were with Jupiter, $42 \%$ with Saturn, $20 \%$ with Neptune, and $11 \%$ with Uranus.

As expected, the same pattern is obtained when we restrict our analysis to the total of extreme close encounters, i.e., encounters occurring within $1 \leqslant r_{\text {td }} \leqslant 10$ (Tables 2 and 3). We found for the forward integrations that four clones of Chariklo were ejected after an extreme close encounter with Jupiter, three with Saturn, and none with Uranus or Neptune. For the backward integrations, we registered 11 clones ejected after a close encounter with Jupiter, five with Saturn, two with Uranus, and one with Neptune.

Among these encounters, we found that only in six cases was Chariklo ejected by just the close encounter that disrupted the rings. For the forward integrations such an event occurred twice an encounter with Jupiter and once after an encounter with Saturn. For the backward integrations we registered two cases with Jupiter and one with Uranus. Thus, our results indicate that for both backward and forward integrations, Jupiter and Saturn are mainly responsible for inserting and removing Chariklo to/from the Centaur region.

However, it is important to point out that since our ejection criterion requires that the object goes beyond $100 \mathrm{AU}$, it is more likely for ejections to be caused by stronger close encounters such as those produced by Jupiter and Saturn. Another consequence of our ejection criterion is that there are bodies that became Trans-Neptunian Objects (TNOs) without being considered as ejected. For example, a Plutino-like object with $a=39 \mathrm{AU}$ and $e=0.2$ would remain a Centaur in our simulations. In any case, regardless of the route that Chariklo took to enter or exit the Centaur region, our results allow us to conclude that it may have brought the rings with it when it was introduced into this region and that it may keep them when it leaves.

\section{FINAL COMMENTS}

In the present work we explored the dynamics of the Centaur 10199 Chariklo and the stability of its rings when disturbed by frequent close encounters with the giant planets. Through numerical integrations we analyzed the orbital evolution of Chariklo as a Centaur (orbiting mainly in the region between Jupiter and Neptune with $a \leqslant 50 \mathrm{AU})$. To do so we considered a sample of 729 clones of Chariklo with small deviations of the semimajor axis, eccentricity, and inclination.

Throughout the lifetime of Chariklo in the population of the Centaurs, we found that close encounters within 1 Hill radius with Uranus are more frequent than encounters with the other planets. Nevertheless, if we look at the most significant close encounters, i.e., those able to disturb or disrupt the rings of Chariklo, we found that Jupiter and Saturn dominate.

For the forward integrations the most significative encounters happen during the last stages of Chariklo among the giant planets, and these occurred exclusively with Jupiter and Saturn. This indicates that Uranus and Neptune may not play an important role in the future dynamics of the rings of Chariklo, but these planets may have had more influence on the rings in the past. For the backward integrations we found a few cases of catastrophic encounters of Chariklo with these planets, three being catastrophic encounters with Uranus and one with Neptune. Nevertheless, the differences between the forward and backward integrations are subtle and probably arose from a statistical analysis based on a small number of extreme close encounters.

In total, for both backward and forward integrations, we found that the number of close encounters able to completely disrupt the rings of Chariklo is small ( $\approx 3 \%$ of the clones), and they most probably occur in the beginning or in the final stages of Chariklo's time as a Centaur. Thus, although a typical Centaur such as Chariklo presents a chaotic orbital evolution (Horner et al. 2004a), we found that if the object had the rings before becoming a Centaur or acquired them in the region of the giant planets, then these rings will probably survive throughout its Centaur life. Hence, the formation of the rings of Chariklo while it has been a Centaur is not mandatory. Therefore, our major conclusion is that Centaurs experience a propitious environment for the existence of rings. Furthermore, if there is an efficient mechanism that creates the rings, then these structures may be common among the bodies of this population. One possible mechanism for the creation rings around Centaurs could be as an outcome of the extreme close encounters with the giant planets studied here. This possibility will be analyzed in a future work.

This work was funded by CAPES, CNPq, and FAPESP (proc. 2011/08171-3). This support is gratefully acknowledged. The authors are also grateful to the PROPE-UNESP for the financial support, and also to the anonymous referee for suggestions that significantly improved the paper.

\section{REFERENCES}

Araujo, R. A. N., \& Winter, O. C. 2014, A\&A, 566, A23

Araujo, R. A. N., Winter, O. C., Prado, A. F. B. A., \& Vieira Martins, R. 2008, MNRAS, 391, 675 
Braga-Ribas, F., Sicardy, B., Ortiz, J. L., et al. 2014, Natur, 508, 72

Brasser, R., Schwamb, M. E., Lykawka, P. S., \& Gomes, R. S. 2012, MNRAS, 420, 3396

Chambers, J. E. 1999, MNRAS, 304, 793

Duffard, R., Pinilla-Alonso, N., Santos-Sanz, P., et al. 2014, A\&A, 564, A92

Emel'Yanenko, V. V., Asher, D. J., \& Bailey, M. E. 2007, MNRAS, 381, 779

Emel'Yanenko, V. V., Asher, D. J., \& Bailey, M. E. 2013, EM\&P, 110, 105

Everhart, E. 1985, in In Dynamics of Comets: Their Origin and Evolution, ed. A. Carusi Carusi, \& G. B. Valsecchi (D. Reidel Publishing Company)

Horner, J., Evans, N. W., \& Bailey, M. E. 2004a, MNRAS, 354, 798
Horner, J., Evans, N. W., \& Bailey, M. E. 2004b, MNRAS, 355, 321

Horner, J., Evans, N. W., Bailey, M. E., \& Asher, D. J. 2003, MNRAS, 343, 1057

Kowal, C. T., Liller, W., \& Marsden, B. G. 1979, in Proc. Symp., The Discovery and Orbit of 2060 Chiron. In: Dynamics of the Solar System (Dordrecht: D. Reidel Publishing Company), 245

Levison, H. F., \& Duncan, M. J. 1997, Icar, 127, 13

Ortiz, J. L., Duffard, R., Pinilla-Alonso, N., et al. 2015, A\&A, 576, A18

Philpott, C. M., Hamilton, D. P., \& Agnor, C. B. 2010, Icar, 208, 824

Sisto, R. P., \& Brunini, A. 2007, Icar, 190, 224

Tiscareno, M. S., \& Malhotra, R. 2003, AJ, 126, 3122 